\title{
Radiation-Induced Graft Polymerization of 4-Vinylpyridine on Polyethylene
}

\author{
A.M. RABIE, ${ }^{*}$ M.I. Aly, ${ }^{* *}$ S.A. Hegazy, ${ }^{* *}$ and \\ N.I. EL-AWADY*** \\ ${ }^{*}$ Chemistry Department, Faculty of Science, \\ Ain Shams University, Cairo, Egypt. \\ **National Centre for Radiation Research and Technology, \\ Madinet Nasr, Cairo, Egypt. \\ ***Laboratory of Polymers and Pigments, National Research Centre, \\ Dokki, Cairo, Egypt.
}

(Received July 7, 1978)

\begin{abstract}
Vinylpyridine was grafted on polyethylene films of different thicknesses by the direct irradiation method using a Co-60 $\gamma$-irradiation source. Addition of different amounts of solvents such as methyl ethyl ketone and cyclohexanone was found to decrease the swelling and the grafting extent on polyethylene. The dependence of the rate of graft polymerization on the thickness of the films was studied. The rate of grafting was found to decrease with increasing film thickness and a first order dependence was obtained. The effect of radiation dose on some of the mechanical properties of the ungrafted and grafted polyethylene was examined. It was found that low doses cause an increase in elongation due to a degradation process of polyethylene chains. At high doses, elongation tends to decrease again due to crosslinking, while the tensile load is not affected. Low grafting percents of 4-vinylpyridine on polyethylene lead to a marked decrease in elongation of the grafted polymer, with almost no change in the tensile load. Increasing the extent of grafting causes a significant increase in the tensile load and almost the same decrease in elongation as for the low grafted polymer.
\end{abstract}

KEY WORDS Grafting / Solvent Effect / Thickness Effect / Tensile Load / Co-60 / $\gamma$-Irradiation /

Radiation-induced graft polymerization of vinylpyridine $^{1}$ on preirradiated polyethylene using $\gamma$ radiation and accelerated electrons has been proved to proceed by radical mechanism with a considerable rate. The grafting rate was dependent on the concentration of the polymer radicals (up to $5 \mathrm{Mrad}$ dose) and on the concentration of the monomer. It was reported also that diffusion plays a certain role on the grafting rate, as the latter is dependent on the thickness of the polyethylene films. Several authors $^{2-6}$ studied the radiation-induced graft polymerization of 4-vinylpyridine onto different polymeric substrates to improve their properties: such as dyeability, ion exchange capability, and capacity to prevent blood coagulation.

Graft polymerization of 4-vinylpyridine on polyethylene, or other polymeric substrates, is not a fully studied system. Little attention has been given to studying the effect of solvents and film thickness (diffusion of monomer into the polymer) on the extent and rate of graft polymerization.

The aim of the present work is to study the effect of the addition of different solvents, such as methyl ethyl ketone and cyclohexanone, on the swelling and the grafting extent of 4-vinylpyridine to polyethylene. The dependence of the graft polymerization rate on the film thickness was also examined by kinetic methods. We also tried to characterize mechanically the behaviour of the grafted and ungrafted polymers by investigating the effect of dose on the elongation and the tensile load of the grafted and the ungrafted polyethylene samples. The apparent and true grafting were also determined.

\section{EXPERIMENTAL}

\section{Materials}

4-Vinylpyridine produced by Fluka AG Chem- 
ische Fabric, Switzerland, of purity $95 \%$ was used with no further purification; methyl ethyl ketone, was a product of Kochlight Laboratories Ltd., England, bp $79-81^{\circ} \mathrm{C}$; methyl alcohol was a product of El-Nasr Company for Pharmaceutical Chemicals, bp $64.5-66.0^{\circ} \mathrm{C}$; cyclohexanone, was a product of Merck, bp $155^{\circ} \mathrm{C}$; xylene was a product of Chemicoke El-Tabin, Egypt, range of boiling $120-150^{\circ} \mathrm{C}$; low-density polyethylene, a product of C.D.F. Chemie. France, in the form of pellets was moulded into sheets by the compression moulding process shown below.

\section{Procedures}

Press Moulding of Polyethylene Sheets. Sheets of polyethylene were prepared according to the following procedure: polyethylene pellets weighing $c a$. $70-75 \mathrm{~g}$ were melted at $200^{\circ} \mathrm{C}$ to blocks of dimensions smaller than the mould. The blocks were sandwiched by aluminium foil, then placed in the mould, and the whole system was heated in the press up to $150-155^{\circ} \mathrm{C}$. When the polyethylene was completely melted, a pressure of $25 \mathrm{~kg} / \mathrm{cm}^{2}$ was applied for $1 \mathrm{~min}$. Then the polyethylene sheet was released from the mould while hot, and the aluminium foil was removed after $2 \mathrm{~h}$. Different mould-thicknesses were used to prepare the grafting films.

Swelling Measurements. The extent of swelling of the polymer was determined by equilibrating known weight samples of polyethylene films with the monomer or the monomer-solvent mixtures at $25^{\circ} \mathrm{C}$ for 4 days. The films were removed, blotted quickly with absorbent paper to remove the surface liquid, and then weighed. The swelling measurements were made on $1.9-\mathrm{mm}$ thick films, due to the higher accuracy attainable with these thicker samples. The problem in using the thinner samples for swelling measurements is that desorption occurs too rapidly during the process of blotting the film surface with the absorbent paper and weighing, leading to relatively large error.

Swelling measurements for polyethylene were made for different concentrations of 4-vinylpyridine in two different solvents, methyl ethyl ketone and cyclohexanone.

Grafting Experiments. Polyethylene films weighing in the range of $0.2-0.5 \mathrm{~g}$ were washed with acetone, dried at $50^{\circ} \mathrm{C}$ under vacuum, weighed, and then immersed in 4-vinylpyridine or 4-vinylpyridinesolvent mixtures in reaction tubes. The polymer- monomer mixtures were equilibrated at $25^{\circ} \mathrm{C}$ for 4 days and then irradiated for specific periods at a dose rate of $74 \mathrm{rad} / \mathrm{s}$ by using a Co- 60 source located at the Regional Centre for Radioactive Isotopes, Cairo. The polyethylene samples were removed from the reaction tubes immediately after irradiation, soaked and washed with methanol for 3 days to clean the film surfaces, and vacuum dried at $40-50^{\circ} \mathrm{C}$ till constant weight was found. The extent of graft polymerization in a sample is calculated as its percentage increase in weight.

$$
\% \text { graft }=\left[\left(w_{t}-w_{i}\right) / w_{i}\right]+100
$$

where $w_{i}$ is the initial weight of the polyethylene sample before irradiation and $w_{t}$ is the weight of the sample after irradiation for time $t$.

Elongation and Tensile Load Measurements. A polyethylene sheet was cut down into a dumbell shape samples with dimensions: 8 -cm length, a neck length of $2.5 \mathrm{~cm}, 4-\mathrm{mm}$ width, and $1.9-\mathrm{mm}$ thickness. The samples were clamped in the jaws of the tensile testing machine type ZP 20, East Germany, in a vertical position. The speed of the moving crosshead was $1 \mathrm{~cm} / \mathrm{min}$. The tensile load and elongation were measured at the break of the polyethylene sample at room temperature.

\section{RESULTS AND DISCUSSION}

\section{Effect of the Solvent on Swelling of Polyethylene}

Cyclohexanone and methyl ethyl ketone were used to study the effect of the addition of a solvent and its concentration on the degree of swelling. Figure 1 shows that addition of methyl ethyl ketone to 4vinylpyridine causes a slight decrease in the degree of swelling of polyethylene films. On the other hand, mixtures of 4-vinylpyridine and cyclohexanone behave differently: an increase in the cyclohexanone content in the monomer-solvent mixture causes a gradual increase in the degree of swelling of polyethylene, reaching a maximum percent swelling for mixtures of $65 \%$ by weight of cyclohexanone. More dilution with cyclohexanone brings about a gradual decrease in the degree of swelling. The high swelling affinity of a cyclohexanone-monomer mixture with $65-\%$ cyclohexanone may be explained by the formation of an isotropic solvent-monomer mixture of a relatively smaller degree of polarity than that of the other mixture compositions and the two components separately. 


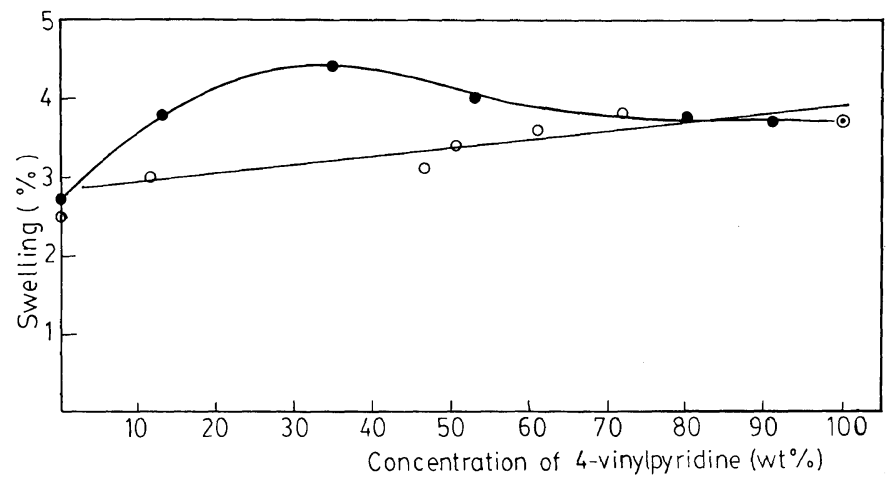

Figure 1. Effect of concentration of 4-vinylpyridine in cyclohexanone $(O)$ and methyl ethyl ketone $(O)$ on the swelling of polyethylene.

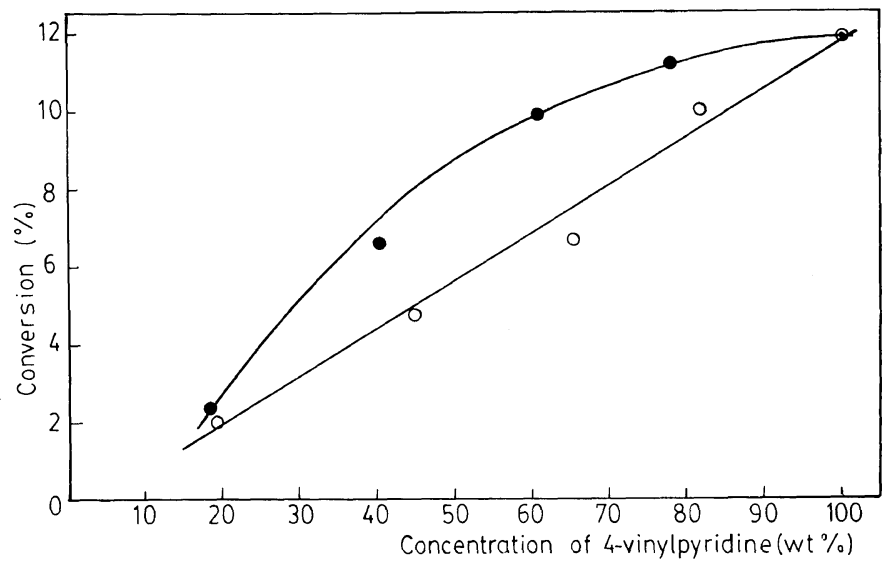

Figure 2. Effect of concentration of 4-vinylpyridine in cyclohexanone $(O)$ and methyl ethyl ketone $(O)$ on the extent of grafting.

\section{Effect of the Solvent on Grafting}

Polyethylene films of 1.9-mm thickness were grafted with different concentrations of 4-vinylpyridine, using cyclohexanone and methyl ethyl ketone as diluents. Grafting was carried out at a dose rate of 74 $\mathrm{rad} / \mathrm{s}$ for $5 \mathrm{~h}$ at $25^{\circ} \mathrm{C}$. Figure 2 shows the effect of the type and concentration of the solvent on the degree of grafting of 4-vinylpyridine on polyethylene. It is shown from the figure that dilutions of the monomer with both solvents cause a decrease in the degree of grafting. A gel effect could be obtained if polymerization is carried out in a highly viscous medium. This medium could be achieved if a poor or non-solvent is used, or if a high degree of conversion is reached.

Cyclohexanone and methyl ethyl ketone were supposed to be precipitating agents for poly(4vinylpyridine) and a gel effect could be expected in the grafting medium. In spite of this prediction, the gel effect was not experimentally verified. This may be partially explained as follows: an increase in the viscosity of the grafting medium due to the solvent effect was partially compensated by its swelling effect on polyethylene and by the power of the monomer to dissolve the grafted poly(4-vinylpyridine) chains.

\section{Effect of Film Thickness on the Grafting Rate}

The effect of film thickness on the rate of graft polymerization was investigated using films of thicknesses $0.36,1.16$, and $1.9 \mathrm{~mm}$. Figure 3 shows the percent graft $v s$. time for the different film thicknesses. From the figure, it is shown that the rate of grafting decreases with increasing film thickness; these results are in agreement with the data found in the literature. ${ }^{7}$ For thick films, the grafting process 


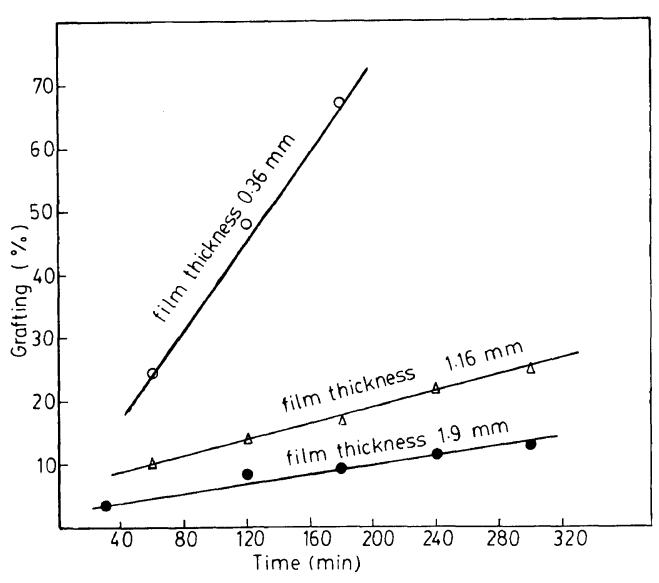

Figure 3. Grafting extent vs. exposure time.

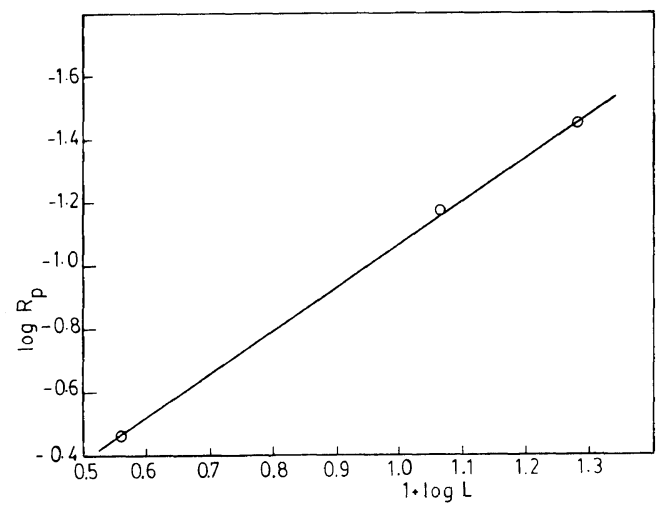

Figure 4. $\log$ (grafting rate) vs. $\log$ (film thickness).

becomes diffusion-controlled and a monomer concentration profile develops within the polymer film thickness. The concentration of monomer is a maximum at the two surfaces and decreases as one moves toward the centre of the film - since monomer diffusion lags behind chemical reaction (polymerization). As diffusion control becomes more extreme, a progressively larger region which is almost devoid of monomer develops in the centre of the film. Therefore, increasing the film thickness brings about more diffusion control and more decrease in the monomer concentration, leading to a pronounced decrease in the rate of grafting.

A plot of $\log$ graft polymerization rate $\left(R_{\mathrm{p}}\right) v s . \log$ film thickness $(L)$, which is shown in Figure 4, gave a straight line with a slope equal to -1.25 . This negative first order dependence of the rate of graft polymerization on the film thickness is in close agreement with previous results. ${ }^{7}$

\section{Apparent and True Grafting}

The apparent graft percentage was calculated as the percent increase in weight of the grafted polyethylene films of $1.9-\mathrm{mm}$ thickness relative to its weight before grafting. For determining the true grafting percent, the grafted films were subjected to an extracting process using xylene as a solvent for the ungrafted polyethylene. The remained poly(ethylene-g-4-vinylpyridine) was subjected again to extraction with methanol to remove the occluded homopoly(4-vinylpyridine), dried, and weighed. The process was repeated till constant weights were attained. The true grafting percentage was then calculated on the basis of the grafted unextractable polyethylene remaining after both extractions. Table I shows the true and apparent grafting percentages; it also shows that the true grafting is much higher than the apparent grafting.

\section{Mechanical Properties of Irradiated and Grafted Polyethylene}

The radiation effect on the mechanical properties of low density polyethylene has been reported previously. ${ }^{8}$ It was found that, upon irradiation of polyethylene, elongation increases slightly up to 10 Mrad and then decreases sharply. Rieke ${ }^{9}$ studied the mechanical properties of the acrylic acid grafted

Table I. Apparent and true grafting

\begin{tabular}{|c|c|c|c|c|c|c|}
\hline $\begin{array}{c}\text { Sample } \\
\text { No. }\end{array}$ & $w_{i}^{\mathrm{a}}$ & $w_{t}^{\mathrm{b}}$ & $w_{t}-w_{i}$ & $\begin{array}{c}\text { Apparent } \\
\text { grafting, } \\
\%\end{array}$ & $w_{\mathrm{e}}{ }^{\mathrm{c}}$ & $\begin{array}{c}\text { True } \\
\text { grafting, } \\
\%\end{array}$ \\
\hline 1 & 0.217 & 0.246 & 0.029 & 13.4 & 0.0365 & 79.5 \\
\hline 2 & 0.121 & 0.137 & 0.016 & 13.2 & 0.0195 & 82.1 \\
\hline 3 & 0.135 & 0.152 & 0.017 & 12.6 & 0.0195 & 87.2 \\
\hline
\end{tabular}

a $w_{i}$, weight of film before grafting; $w_{t}$, weight of film after grafting; $w_{\mathrm{e}}$, weight of an extracted grafted film. 


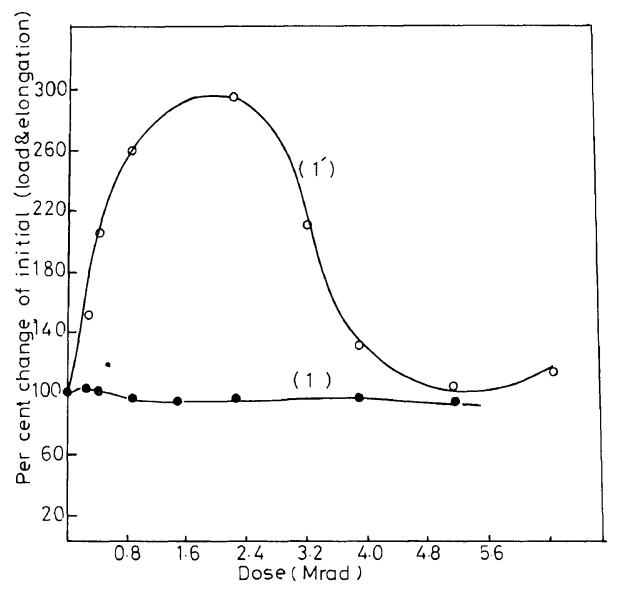

Figure 5. Change of tensile load and elongation per cent of initial with irradiation dose for ungrafted polyethylene.

polyethylene and found that the tensile moduli follow the characteristic pattern observed when a rigid filler is added to a flexible polymer.

Curves $1 \& 1^{\prime}$ in Figure 5 show the percent change (compared to the unirradiated polyethylene) of the tensile load and elongation, respectively, as a function of radiation dose, for the ungrafted polyethylene. It is clear that the irradiation to a dose of ca. $2.4 \mathrm{Mrad}$ causes a marked increase in elongation (the first part of the curve $1^{\prime}$ ). Increasing the radiation dose higher than 2.4 Mrads decreases the elongation percent (the second part of curve 1'). The increase in elongation may be due to a degradation process, while the decrease in elongation could be attributed to a crosslinking mechanism. Oxidation of polyethylene during irradiation is mentioned in the literature ${ }^{10}$ Oxidation may induce a slight degradation in the main chain of the polyethylene; after most of the trapped oxygens were consumed, crosslinking of the chains dominates over degradation and consequently elongation decreases. It seems that degradation or crosslinking of the polymer chains at these low doses is not sufficient to influence the tensile load, while it affects the elongation to a great extent.

Investigation of the mechanical properties for grafted polyethylene was carried out for highly grafted and low grafted polyethylene for two film thicknesses, 0.05 and $1.9 \mathrm{~mm}$. Table II gives the percent graft for the two film thicknesses at different doses. From the table, it is obvious that the percent
Table II. Effect of radiation dose on grafting yield

\begin{tabular}{ccc}
\hline & \multicolumn{2}{c}{ Grafting yield percent } \\
\cline { 2 - 3 } & $\begin{array}{c}1.9-\mathrm{mm} \\
\text { polyethylene } \\
\text { film }\end{array}$ & $\begin{array}{c}\text { 0.05-mm } \\
\text { polyethylene } \\
\text { film }\end{array}$ \\
\hline 0.14 & 3.6 & 29.2 \\
0.27 & - & 76.7 \\
0.54 & 8.4 & 79.2 \\
0.81 & 9.6 & 133.3 \\
1.08 & 11.4 & 188.3 \\
1.35 & 12.9 & 239.2 \\
\hline
\end{tabular}

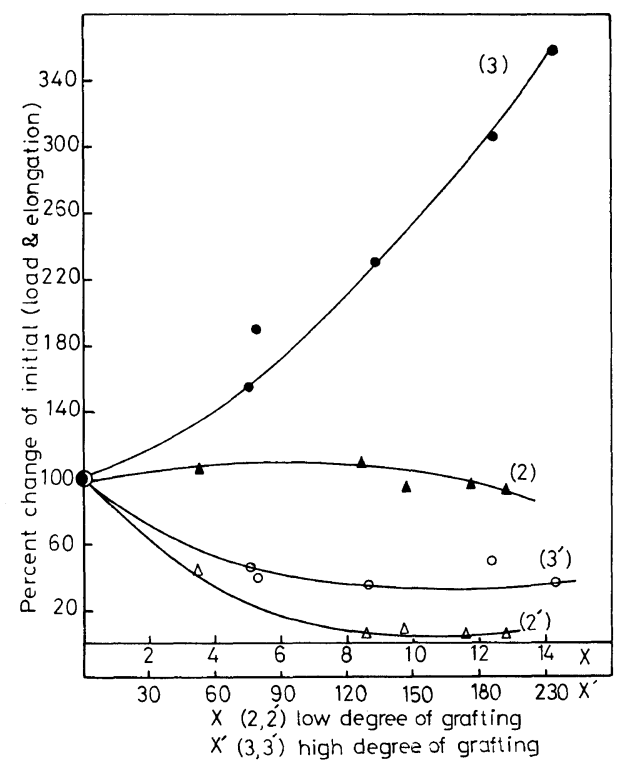

Figure 6. Dependence of tensile load and elongation per cent from initial on the degree of grafting.

graft for thin films is higher than that for thicker ones for the same dose. The effect of low grafting percentage is more highly pronounced on elongation (curve $2^{\prime}$ ) than on the tensile load (curve 2) as shown in Figure 6. The high grafting ratios still affect the elongation (curve $3^{\prime}$ ) in the same magnitude as for low grafted films (curve $2^{\prime}$ ), but cause a remarkable increase in the tensile load (curve 3) for highly grafted films. This behaviour may be explained by the model of a flexible polymer filled with a rigid filler, namely poly(4-vinylpyridine).

Acknowledgment. This work had been carried out in the laboratories of the National Research 
Centre, Dokki, and the National Centre for Radiation Research and Technology. Thanks are due to Prof. Dr. N.A. Ghanem, head of the laboratory of polymers and pigments, for his keen interest and support. This work is the main part of the Master Degree in Science of Mr. El-Sayed Ahmed Abd El-Aziz Hegazy.

\section{REFERENCES}

1. L. Sawtschenko and K. Jobst, Plaste Kautsch., 16, 891 (1969).

2. T. Okada, Y. Suzuki, K. Kohedera, and I. Sakurada, JAERI Report, 5018, Vol. 5, 1968; A. Danno, At. Energ. Rev., 2, 399 (1971).

3. M. R. Houlton and J. K. Thomas, Int. Appl. Radiat. Isot., 11, 45 (1961).
4. W. Zielinski and T. Achmatowicz, Institute of Nuclear Research, Warsaw, No. 490/XVII (1963); Chem. Abstr., 61, 10828 (1964).

5. G. W. Stanton and T. G. Traylor, U. S. Patent 3, 048,561 Aug. 7, 1962, Chem. Abstr., 57, 14020 (1962).

6. R. D. Falb, G. A. Crode, M. M. Epstein, B. G. Brand, and R. L. Leninger, Summary Report on "Development of Blood-Compatible Polymeric Materials" from Battle Memorial Institute, 1965, June 29.

7. G. Odian, R. Henery, R. Koenig, D. Mangaraj, L. D. Trung, B. Chao, and A. Derman, J. Polym. Sci., Polym. Chem. Ed., 13, 623 (1975).

8. A. Charlesby, "Atomic Radiation and Pólymers," Pergamon Press Ltd., London, 1960, p. 349.

9. J. K. Rieke, G. M. Hart, and F. L. Saunders, J. Polym. Sci., Part C, 4, 589 (1964).

10. A. Charlesby, "Atomic Radiation and Polymers," Pergamon Press Ltd., London, 1960, p. 236. 